\title{
A SEPARATION THEOREM FOR EXPECTED VALUE AND FEARED VALUE DISCRETE TIME CONTROL
}

\author{
PIERRE BERNHARD
}

\begin{abstract}
We show how the use of a parallel between the ordinary $(+, \times)$ and the $(\max ,+)$ algebras, Maslov measures that exploit this parallel, and more specifically their specialization to probabilities and the corresponding cost measures of Quadrat, offer a completely parallel treatment of stochastic and minimax control of disturbed nonlinear discrete time systems with partial information. This paper is based upon, and improves, the discrete time part of the earlier paper [9].
\end{abstract}

\section{INTRODUCTION}

Minimax control, or worst case design, as a means of dealing with uncertainty is an old idea. It has gained a new popularity with the recognition, in 1988 , of the fact that $H_{\infty}$ optimal control could be cast into that concept. Although some work in that direction existed long before (see [10]), this viewpoint has vastly renewed the topic. See [4] and related work.

Many have tried to extend this work to a nonlinear setup. Most prominent among them perhaps is the work of Isidori, $[16,17]$ but many others have followed suit : $[27,5,6]$ and more recently $[19,20,21]$. This has contributed to a renewed interest in nonlinear minimax control.

If we decide that minimax is an alternative to stochastic treatment of disturbances, it makes sense to try to establish a parallel. To that end, we build upon the work of Maslov and coworkers [23, 22, 24] on Maslov measures, revisiting in fact Choquet's capacities [12] and more specifically on the work of Quadrat and coworkers [25, 3, 2, 1] who developped the dual in that parallel to probability theory, introducing a special class of Maslov measures, cost measures. One can also refer to the work of Del Moral [14, 15]

Our main aim is to stress how the use of these tools allows one to give a treatment of minimax control completely parallel to that of stochastic control, introducing the mathematical fear as a parallel to mathematical expectation. As an example, we develop two parallel separation theorems, none really new: the first (stochastic) is probably rather classical, and can be found at least in (manuscript) notes by Michel Viot [28], and the second (minimax) is a generalization of results of Whittle [29], and ourselves [8, 4]. The case without separation principle is a generalization of our work [7]

INRIA Sophia Antipolis, 2004, route des Lucioles, B.P. 93, 06902 Sophia Antipolis Cedex, France.

Received by the journal January 24, 1996. Accepted for publication April 29, 1996. 
and of [21]. The difference with these references, as well as with [4], or [9] where we introduced the wording "mathematical fear", is in the way the result is derived, and specifically the substitution of a cost measure to the "informational state" of [21] or the cost to go of [4] or the "stress" of [29]. It is thanks to that mild modification that we get a complete parallel.

An example of the use of (a variable end time version of) the theory of this paper in a nonlinear minimax problem (actually continuous time), both with and without the conditions for the separation theorem to apply holding, can be found in [26].

\section{QUADRAT'S MORPHISM}

In a series of papers [25, 3, 2], Quadrat and coauthors have fully taken advantage of the "morphism" introduced between the ordinary algebra $(+, x)$ and the $(\min ,+)$, or alternatively the $(\max ,+)$, algebra to develop a $d e$ cision calculus parallel to probability calculus. It has been pointed out by Quadrat and coauthors that a possible way of understanding that morphism was through Cramer's transform. We shall not, however, develop that way of thinking here, but merely rely on the algebraic similarity between the two calculus, as stressed by the theory of Maslov measures.

Let us briefly review some concepts, based on [2].

\subsection{Cost measure}

The parallel to a probability measure is a cost measure. Let $\Omega$ be a topological space, $\mathcal{A}$ a family of open subsets, $K: \mathcal{A} \rightarrow \mathbb{R} \cup\{-\infty\}$ is called a cost measure if it satisfies the following axioms :

- $K(\emptyset)=-\infty$

- $K(\Omega)=0$

- for any family of elements $A_{n}$ of $\mathcal{A}$,

$$
K\left(\cup A_{n}\right)=\sup _{n} K\left(A_{n}\right) .
$$

(Notice that the probabilist equivalent of the above formula, with a sum, holds only for disjoint subsets. However, we could state the axiom with that restriction, the above formula would automatically hold for any family.)

The first and third axioms are that of Maslov measures. The second one specializes it as a cost measure. One may notice the parallel with a probability measure. In the first two axioms, the 0 of probability measures, the neutral element of the addition, is replaced by the neutral element of the max operator : $-\infty$, and the 1 , the neutral element of the product, is replaced by the neutral element of the sum, 0 . In the third axiom, the sum of the measures of the disjoint sets is replaced by the max.

In the theory of Maslov measures, the habit is to denote the max as $\oplus$, the sum as a product, $-\infty$ as $(0), 0$ as $\mathbb{1 1}$ so that $a \oplus(0)=a$, and $a \cdot \mathbb{1}=a$. However, one has $a \oplus a=a$ so that this school refers to idempotent algebra and idempotent measures. This leads to a theory of integration which is 
equivalent to the penalized supremums that we shall use in the minimax theory below, but stressing further the parallel with classical measures and integration. This parallel continues with the following concept:

The function $\Gamma: \Omega \rightarrow \mathbb{R} \cup\{-\infty\}$ is called a cost density of $K$ if we have

$$
\forall A \in \mathcal{A}, \quad K(A)=\sup _{\omega \in \Omega} \Gamma(\omega) .
$$

One has the following theorem (Kolokoltsov and Maslov [22], Akian [1]) : Proposition 2.1. Every cost measure defined on the open sets of a Polish space $\Omega$ admits a unique maximal extension to $2^{\Omega}$, this extension has a density, which is a u.s.c. function.

\subsection{Feared VALUE}

The wording Feared value ${ }^{1}$ is used here to stress the parallel with expected value.

When a stochastic disturbance is introduced into a problem model, in order to derive a controller design for instance, it comes with a given probability distribution. We shall always assume here that these distributions have densities. Let therefore $w \in \mathrm{W}$ be a stochastic variable. Let its probability density be $\Pi(\cdot)$. Let $\psi$ be a function of $w$ with values in $\mathbb{R}$. We define its expected value as

$$
\mathbb{E}_{w} \psi:=\int \psi(w) \Pi(w) d w
$$

and we omit the subscript $w$ to $\mathbb{E}$ when no ambiguity results.

Similarly, let a disturbance $w$ be given together with a cost density $\Gamma(\cdot)$. The feared value of a function $\psi$ from $W$ into $\mathbb{R}$ is defined as

$$
\mathbb{F}_{w} \psi:=\max _{w}[\psi(w)+\Gamma(w)]
$$

which is the formula dual to that of the expected value in Quadrat's morphism.

The "Fear" operator enjoys the linearity properties one would expect in the $(\max ,+)$ algebra :

$$
\mathbb{F}(\max \{\phi, \psi\})=\max \{\mathbb{F} \phi, \mathbb{F} \psi\},
$$

and if $\lambda$ is a constant,

$$
\mathbb{F}(\lambda+\psi)=\lambda+\mathbb{F} \psi
$$

INDUCED MEASURE. Exactly as a measurable function from a probability space into $\mathbb{R}^{n}$ is called a (vector) random variable, a continuous function from a cost measured space into $\mathbb{R}^{n}$ is called a (vector) decision variable. The cost measure of the underlying space induces a cost measure on the decision variable in a natural way.

Let a decision variable $x$ have a cost density $C(x)$. It is convenient here to assume it has a compact domain. Let $y=h(x)$, say with $h$ continuous.

\footnotetext{
${ }^{1}$ In French Frayeur mathématique parallel to Espérance mathématique
} 
Then, $y$ is itself a decision variable, with an induced cost measure that can be deduced from the inspection of the feared value of an arbitrary function $\phi(y)$ :

$$
\mathbb{F} \phi=\max _{x}[\phi(h(x))+C(x)]=\max _{y}\left[\phi(y)+\max _{x \in h^{-1}(y)} C(x)\right] .
$$

Hence we conclude that the cost density of $y$ is

$$
D(y)=\max _{x \in h^{-1}(y)} C(x)
$$

Observe that this is indeed a cost density, since it is normalized:

$$
\max _{y} D(y)=\max _{x} C(x)=0 .
$$

Conditioning. We may introduce the conditioning by an event. ${ }^{2}$ Let $v \in \mathrm{V}$ be a decision variable, $B \subset \mathrm{V}$ be a subset of $\mathrm{V}$, and as usual $K(B)=$ $\max _{v \in B} \Gamma(v)$. Let

$$
\Gamma(v \mid B)= \begin{cases}\Gamma(v)-K(B) & \text { if } v \in B, \\ -\infty & \text { if } v \notin B .\end{cases}
$$

This is the "natural" cost density on $v$ if it is constrained to belong to $B$.

This yields

$$
K(A \mid B):=\max _{v \in A} \Gamma(v \mid B)=\max _{v \in A \cap B} \Gamma(v)-K(B),
$$

leading to the counterpart of Bayes formula:

$$
K(A \cap B)=K(A \mid B)+K(B) .
$$

Let now a pair of decision variables $(v, w)$ ranging over sets $\mathrm{V} \times \mathrm{W}$ have a joint cost density $\Gamma(v, w)$. We may define the marginal laws $\Gamma_{1}$ and $\Gamma_{2}$ for $v$ and $w$ respectively as

$$
\Gamma_{1}(v)=\max _{w \in \mathbb{W}} \Gamma(v, w), \quad \Gamma_{2}(w)=\max _{v \in \mathrm{V}} \Gamma(v, w) .
$$

Let $K$ denote the cost measure generated by $\Gamma$ on $\mathrm{V} \times \mathrm{W}$. One has for instance, for a subset $A \subset \mathrm{V}, K(A \times \mathrm{W})=\mathbb{F}_{v}^{\Gamma_{1}}\left(\mathbb{1}_{A}(v)\right)$. Here, the symbol $\mathbb{F}_{v}^{\Gamma_{1}}$ means Feared value with respect to $v$ endowed with the cost density $\Gamma_{1}$, and $\mathbb{1}_{A}$ is the characteristic function of $A$, equal to 0 if $v \in A$ and to $-\infty$ otherwise.

We now apply the previous concept of conditioning to the cylindrical condition set $B=\mathrm{V} \times\{w\}$. This leads to the conditional cost density

$$
\Gamma(v \mid w)=\Gamma(v, w)-\Gamma_{2}(w)
$$

and notice that this is indeed a cost density (i.e. normalized). The decision variables $v$ and $w$ are said to be independant if $\Gamma(v \mid w)=\Gamma_{1}(v)$, which is thus, as expected, equivalent to $\Gamma(v, w)=\Gamma_{1}(v)+\Gamma_{2}(w)$.

\footnotetext{
${ }^{2}$ This is a "naive" theory of conditioning, based upon Bayes rule. For an equivalent of the full stochastic theory of conditional expectations based upon a sigma-field representation, see Del Moral [14, 15]

Esaim: CoCv, July 1996, Vol. 1, Pr. 191-206
} 
A sequence of stochastic variables or stochastic process $\left\{w_{t}\right\}, t=0 \ldots T-$ 1 also denoted $w_{[0, T-1]}$, are independant, or constitute a white stochastic process, if their joint probability density is the product of their individual probability densities $\Pi_{t}$ :

$$
\Pi\left(w_{[0, T-1]}\right)=\prod_{t=0}^{T-1} \Pi_{t}\left(w_{t}\right)
$$

leading to the following formula, where $J$ is a function of the whole sequence

$$
\mathbb{E} J\left(w_{[0, T-1]}\right)=\int J\left(w_{[0, T-1]}\right) \prod_{t=0}^{T-1} \Pi_{t}\left(w_{t}\right) d w_{[0, T-1]} .
$$

In a similar fashion, a sequence of independant decision variables $w_{[0, T-1]}$, or white decision process with cost densities $\Gamma_{t}$ will have a joint cost density $\Gamma$ equal to the sum of their individual cost densities:

$$
\Gamma\left(w_{[0, T-1]}\right)=\sum_{t=0}^{T-1} \Gamma_{t}\left(w_{t}\right)
$$

leading to the dual formula

$$
\mathbb{F} J\left(w_{[0, T-1]}\right)=\max _{w_{[0, T-1]}}\left[J\left(w_{[0, T-1]}\right)+\sum_{t=0}^{T} \Gamma_{t}\left(w_{t}\right)\right] .
$$

\section{THE DiscreTE TIME CONTROL PROBLEM}

We consider a partially observed two input control system

$$
\begin{aligned}
x_{t+1} & =f_{t}\left(x_{t}, u_{t}, w_{t}\right), \\
y_{t} & =h_{t}\left(x_{t}, w_{t}\right),
\end{aligned}
$$

where $x_{t} \in \mathbb{R}^{n}$ is the state at time $t, u_{t} \in \mathrm{U}$ the (minimizer's) control, $w_{t} \in \mathrm{W}$ the disturbance input, and $y_{t} \in \mathrm{Y}$ the measured output. We shall call $\mathbf{U}$ the set of in put sequences over the time horizon $[0, T-1]:\left\{u_{t}\right\}_{t \in[0, T-1]}$ usually written as $u_{[0, T-1]} \in \mathbf{U}$, and likewise for $w_{[0, T-1]} \in \mathbf{W}$. The initial state $x_{0} \in \mathrm{X}_{0}$ is also considered part of the disturbance. We shall call $\omega=\left(x_{0}, w_{[0, T-1]}\right)$ the combined disturbance, and $\Omega=\mathrm{X}_{0} \times \mathbf{W}$ the set of disturbances.

The solution of (2) (3) above shall be written as

$$
\begin{aligned}
x_{t} & =\phi_{t}\left(u_{[0, T-1]}, \omega\right), \\
y_{t} & =\eta_{t}\left(u_{[0, T-1]}, \omega\right) .
\end{aligned}
$$

Finally, we shall call $u^{t}$ a partial sequence $\left(u_{0}, u_{1}, \ldots, u_{t}\right)$ and $\mathrm{U}^{t}$ the set of such sequences ${ }^{3}$, likewise for $w^{t} \in \mathrm{W}^{t}$ and $y^{t} \in \mathrm{Y}^{t}$. Also, we write $\omega^{t}=\left(x_{0}, w^{t}\right) \in \mathrm{X}_{0} \times \mathbf{W}^{t}=: \Omega^{t}$.

\footnotetext{
${ }^{3}$ notice the slight inconsistency in notations, in that our $\mathrm{U}^{t}$ is the cartesian $(t+1)$ power of $\mathrm{U}$. Other choices of notations have their drawbacks too.
} 
The solution of (2) and (3) may alternatively be written as

$$
\begin{aligned}
x_{t} & =\phi_{t}\left(u^{t-1}, \omega^{t-1}\right), \\
y_{t} & =\eta_{t}\left(u^{t-1}, \omega^{t}\right) .
\end{aligned}
$$

We shall also write

$$
\begin{aligned}
x^{t} & =\phi^{t}\left(u^{t-1}, \omega^{t-1}\right), \\
y^{t} & =\eta^{t}\left(u^{t-1}, \omega^{t}\right),
\end{aligned}
$$

to refer to the partial sequences solution of (2) and (3)

Admissible controllers will be strictly causal output feedbacks of the form $u_{t}=\mu_{t}\left(u^{t-1}, y^{t-1}\right)$. We denote by $\mathcal{M}$ the class of such controllers.

A performance index is given. In general, it may be of the form

$$
J\left(x_{0}, u_{[0, T-1]}, w_{[0, T-1]}\right)=M\left(x_{T}\right)+\sum_{t=0}^{T-1} L_{t}\left(x_{t}, u_{t}, w_{t}\right) .
$$

However, we know that, to the expense of increasing the state dimension by one if necessary, we can always bring it back to a purely terminal payoff of the form

$$
J\left(x_{0}, u_{[0, T-1]}, w_{[0, T-1]}\right)=M\left(x_{T}\right)=M \circ \phi_{T}\left(u_{[0, T-1]}, \omega\right) .
$$

The data of a strategy $\mu \in \mathcal{M}$ and of a disturbance $\omega \in \Omega$ generates through (2) (3) a unique pair of sequences $\left(u_{[0, T-1]}, w_{[0, T-1]}\right) \in \mathbf{U} \times \mathbf{W}$. Thus, with no ambiguity, we may also use the abusive notation $J(\mu, \omega)$. The aim of the control is to minimize $J$, in some sense, "in spite of the unpredictable disturbances".

We want to compare here two ways of turning this unprecise statement into a meaningful mathematical problem.

Stochastic CONTRol. In the first approach, stochastic control, we modelize the unknown disturbance as a random variable, more specifically here a random variable $x_{0}$ with a probability density $P_{0}(x)$ and an independant white stochastic process $w_{[0, T-1]}$ of known instantaneous probability distribution $\Pi_{t}$. (We notice that nothing in the sequel prevents $\Pi_{t}$ from depending on $x_{t}$ and $u_{t}$.) The criterion to be minimized is then

$$
G(\mu):=\mathbb{E}_{\omega} J(\mu, \omega) .
$$

This can be expanded into

$$
G(\mu)=\int M\left(x_{T}\right)\left(\prod_{t=0}^{T-1} \Pi_{t}\left(w_{t}\right)\right) P_{0}(\xi) d w_{[0, T-1]} d \xi
$$

This can be characterized as a weighted average since the perturbation values are weighted through their probability density.

Minimax CONTROL. In the second approach, we are given the cost density $Q_{0}$ of $x_{0}$, and the cost densities $\Gamma_{t}$ of the $w_{t}$ 's. (Again, $\Gamma_{t}$ might depend on Esaim: CoCv, JULy 1996, Vol. 1, Pr. 191-206 
$x_{t}$ and $u_{t}$.) The criterion to be minimized is then

$$
H(\mu):=\mathbb{F}_{\omega} J(\mu, \omega),
$$

which can be expanded into

$$
H(\mu):=\max _{w_{[0, T-1]}} \max _{\xi}\left[M\left(x_{T}\right)+\sum_{t=0}^{T-1} \Gamma_{t}\left(w_{t}\right)+Q_{0}(\xi)\right]
$$

This can be characterized as a penalized max, since the perturbation values are penalized through their cost density.

REMARK 3.1. If all cost measures of the disturbances are taken constant, (hence 0 by normalization), then $H(\mu)$ is, if it exists, the guaranteed value given only the sets over which the perturbations range. Therefore, minimizing it is insuring the best possible guaranteed value.

\section{DYNAMIC PROGRAMMING}

\subsection{Stochastic dynamic Programming}

4.1.1. Full information. We quickly recall here for reference purposes the classical solution of the full information problem via dynamic programming.

In the case where the controller has perfect and instantaneous knowledge of the current state, and is therefore allowed to use state feedback control strategies of the form $u_{t}=\varphi_{t}\left(x_{t}\right)$, it is well known that a solution of the optimal control problem is obtained as follows.

We introduce the full information Bellman return function $V_{t}$ defined by the classical dynamic programming recursion :

$$
\begin{gathered}
\forall x \in \mathbb{R}^{n}, \quad V_{T}(x)=M(x), \\
\forall x \in \mathbb{R}^{n}, \quad V_{t}(x)=\inf _{u} \mathbb{E}_{w_{t}} V_{t+1}\left(f_{t}\left(x, u, w_{t}\right)\right) .
\end{gathered}
$$

The infimum of the performance index $G(\varphi)$ is $\mathbb{E}_{x}^{P_{0}} V_{0}(x)$ (where we recall that the probability density $P_{0}$ of $x_{0}$ is a data). Furthermore, if the minimum is reached for all $(t, x)$ in $(12)$, then the argument $\varphi_{t}^{*}(x)$ of the minimum is an optimal strategy.

4.1.2. Partial information. Let us turn now to the (almost as classical) solution of the partial information, stochastic problem. One has to introduce the conditional state probability measure, and, assuming it is absolutely continuous with respect to the Lebesgue measure, its density $P$. Let, thus, $P_{t}(x) d x$ be the conditional probability measure of $x_{t}$ given $y^{t-1}$, or a priori state probability distribution at time $t$, and $P_{t}^{\eta}(x) d x$ be the conditional state distribution given $y^{t-1}$ and given that $y_{t}=\eta$, or a posteriori state probability distribution at time $t$. Notice also that our notations are consistent, in that at time $t=0$, with no information available yet beyound the data of the problem, the probability density of $x$ is $P_{0}$. 
Clearly, $P_{t}$ is a function only of past measurements and controls, assuming these are causal, i.e. independant from future disturbances. As a matter of fact, we can give the filter that lets one compute it. Starting from $P_{0}$, at each step, $P_{t}^{\eta}$ can be obtained by Bayes rule. It suffices here to notice that, because the information is increasing, (the information algebras are nested), we have, for any test function $\psi(\cdot) \in L^{1}\left(\mathbb{R}^{n}\right)$,

$$
\mathbb{E}_{y} \int \psi(x) P_{t}^{y}(x) d x=\int \psi(x) P_{t}(x) d x=: \mathbb{E}_{x}^{P_{t}} \psi(x) .
$$

Then $P_{t+1}$ is obtained by propagating $P_{t}^{y t}$ through the dynamics. It suffices for our purpose to define this propagation by the dual operator: for any test function $\psi$,

$$
\mathbb{E}_{y} \mathbb{E}_{x}^{P_{t+1}} \psi(x)=\mathbb{E}_{x}^{P_{t}} \mathbb{E}_{w}^{\Pi_{t}} \psi\left(f_{t}\left(x, u_{t}, w\right)\right)
$$

The above calculations also emphasize the dependance of the sequence $\left\{P_{t}\right\}$ on the control sequence $u_{[0, T-1]}$ and the observation sequence $y_{[0, T-1]}$. Let this define the function $\mathcal{F}_{t}$ as

$$
P_{t+1}=\mathcal{F}_{t}\left(P_{t}, u_{t}, y_{t}\right) .
$$

Let $\mathcal{P}_{t}$ be the set of all possible such probability distributions $P_{t}$, for all sequences $u_{[0, T-1]}$ and all $\omega$.

Via a standard dynamic programming argument, we can check that the Bellman return function $U$ is obtained by the recurrence relation

$$
\begin{aligned}
\forall P \in \mathcal{P}_{T}, \quad U_{T}(P) & =\mathbb{E}_{x}^{P} M(x), \\
\forall t \in[0, T-1], \forall P \in \mathcal{P}_{t}, \quad U_{t}(P) & =\inf _{u} \mathbb{E}_{y} U_{t+1}\left(\mathcal{F}_{t}(P, u, y)\right) .
\end{aligned}
$$

We can state the following theorem:

THEOREM 4.1. If there exists a sequence of functions $\left\{U_{t}\right\}$ from $\mathcal{P}_{t}$ into $\mathbb{R}$ satisfying equations (16)(17), then the optimal cost is $U_{0}\left(P_{0}\right)$.

Moreover, assume that the minimum in $u$ is attained in (17) above at $u=\hat{\mu}_{t}(P)$. Then (15) and

$$
u_{t}=\hat{\mu}_{t}\left(P_{t}\right)
$$

define an optimal controller for the stochastic control problem.

Proof. Consider a causal sequence of controls $\left\{u_{t}\right\}$ and the associated sequence $\left\{P_{t}\right\}$. (The very definition and calculation procedure of $P_{t}$ implies that $u_{t}$ has to be independant from the future disturbances). Rewrite (17) as

$$
U_{t}\left(P_{t}\right) \leq \mathbb{E}_{y_{t}} U_{t+1}\left(P_{t+1}\right) .
$$

Apply this at time $t=T-1$, using (16) and (14):

$$
U_{T-1}\left(P_{T-1}\right) \leq \mathbb{E}_{x}^{P_{T-1}} \mathbb{E}_{2 w}^{\Pi_{T-1}} M\left(f_{T-1}\left(x, u_{T-1}, w\right)\right) .
$$


We may now iterate:

$$
\begin{aligned}
& U_{T-2}\left(P_{T-2}\right) \leq \\
& \quad \mathbb{E}_{x}^{P_{T-2}} \mathbb{E}_{w_{T-2}}\left[\mathbb{E}_{w_{T-1}} M\left(f_{T-1}\left(f_{T-2}\left(x, u_{T-2}, w_{T-2}\right), u_{T-1}, w_{T-1}\right)\right)\right.
\end{aligned}
$$

and so on until we get

$$
U_{0}\left(P_{0}\right) \leq \mathbb{E}_{x}^{P_{0}} \mathbb{E}_{w_{[0, T-1]}} M \circ \phi_{T}\left(u_{[0, T-1]}, x_{0}, w_{[0, T-1]}\right)
$$

And according to formula (8), the rihgt-hand side obove is just $\mathbb{E} J$. Furthermore, an appropriate choice of $u_{t}$ at each step allows the controller to be arbitrarily close to equality in each of the inequalities, thus establishing $U_{0}\left(P_{0}\right)$ as the infimum of the criterion. Moreover, if the minimizing control (18) exists, all inequalities are equalities, and the infimum of the criterion is reached by that strategy, which is clearly causal, and thus optimal.

\subsection{Minimax Dynamic Programming}

4.2.1. Full information. Let us quickly review Isaacs' dynamic programming theory for the full information problem, i.e. for state feedback strategies.

We introduce the full information Isaacs Value function $V_{t}(x)$ which satisfies the classical Isaacs equation:

$$
\begin{gathered}
\forall x \in \mathbb{R}^{n}, \quad V_{T}(x)=M(x), \\
\forall x \in \mathbb{R}^{n}, \quad V_{t}(x)=\inf _{u} \mathbb{F}_{w_{t}} V_{t+1}\left(f_{t}\left(x, u, w_{t}\right)\right) .
\end{gathered}
$$

Notice that we have an identical formula with that of subsection 4.1.1, simply replacing $\mathbb{E}$ by $\mathbb{F}$.

Notice also that we do not need that the Isaacs condition, i.e. the existence of a saddle point in the right hand side above, hold. If it does not, $V$ is an upper value, which is what is needed in the context of minimax control.

4.2.2. Partial information. The theory reviewed here is a modification of results first published in [21], also derived in [7] in essentially the same way as here.

Let us consider now the problem of minimizing $H(\mu)$. We have to introduce the conditional state cost measure and its cost density $Q$ (according to the concepts introduced in section 2.1 following [2]). It is defined as the normalized maximum possible past cost knowing the past information, as a function of current state. In the previous papers such as [21], [7, 8, 9], [13], the authors used the so-called informational state (James et al.) or cost to go (Başar, Bernhard) which is an un-normalized version of the present conditional state cost density. The result was a lack of symmetry with the stochastic case, and as a result less appealing formulas.

To be more precise, let us introduce the following subsets of $\Omega$. Given a pair $\left(u^{t}, y^{t}\right) \in \mathrm{U}^{t} \times \mathrm{Y}^{t}$, and a subset $A$ of $\mathbb{R}^{n}$, let

$$
\Omega_{t}\left(A \mid u^{t}, y^{t}\right)=\left\{\omega \in \Omega \mid y^{t}=\eta^{t}\left(u^{t-1}, \omega^{t}\right), \text { and } \phi_{t+1}\left(u^{t}, \omega^{t}\right) \in A\right\} .
$$


For any $x \in \mathbb{R}^{n}$, we shall write $\Omega_{t}\left(x \mid u^{t}, y^{t}\right)$, or simply $\Omega_{t}(x)$ when no ambiguity results, for $\Omega_{t}\left(\{x\} \mid u^{t}, y^{t}\right)$. And likewise for $\Omega_{t-1}(x)$.

The "conditional worst cost to go" of $A$ (or the "informational state") is the Maslov measure $\sup _{\omega \in \Omega_{t-1}(A)}\left[Q_{0}\left(x_{0}\right)+\Gamma\left(w_{[0, T-1]}\right)\right]$, and hence the associated Maslov density function is, for $t>0$,

$$
W_{t}(x)=\sup _{\omega \in \Omega_{t-1}(x)}\left[\sum_{k=0}^{T-1} \Gamma_{k}\left(w_{k}\right)+Q_{0}\left(x_{0}\right)\right]=\sup _{\omega \in \Omega_{t-1}(x)}\left[\sum_{k=0}^{t-1} \Gamma_{k}\left(w_{k}\right)+Q_{0}\left(x_{0}\right)\right] .
$$

Initialize this sequence with $W_{0}=Q_{0}$ which constitutes the cost density of $x_{0}$ before any further information is available.

It is a simple matter to write recursive equations of the form

$$
W_{t+1}=\widetilde{\mathcal{G}}_{t}\left(W_{t}, u_{t}, y_{t}\right) .
$$

In fact, $\widetilde{\mathcal{G}}_{t}$ is defined by the following. Let for ease of notations

$$
\mathrm{Z}_{t}(x \mid u, y)=\left\{(\xi, v) \in \mathbb{R}^{n} \times \mathbf{W} \mid f_{t}(\xi, u, v)=x, \quad h_{t}(\xi, v)=y\right\},
$$

then we have

$$
W_{t+1}(x)=\sup _{(\xi, v) \in Z_{t}\left(x \mid u_{t}, y_{t}\right)}\left[W_{t}(\xi)+\Gamma_{t}(v)\right] .
$$

As was probably first shown in [21], (also presented in a talk in Santa Barbara in july 1993), one can do simple dynamic programming in terms of this function $W_{t}$, ranging over the space $\mathcal{W}_{t}$. The value function $U$ will now be obtained through the following relation

$$
\text { (22) } \forall t \in[0, T-1], \forall W \in \mathcal{W}_{t}, \quad U_{t}(W)=\inf _{u} \sup _{y} U_{t+1}\left(\widetilde{\mathcal{G}}_{t}(W, u, y)\right) .
$$

Moreover, assume that the minimum in $u$ is attained in (22) above at $u=$ $\hat{\mu}_{t}(W)$. Then it defines an optimal feedback analogous to (18), with $W_{t}$ now defined by (20), for the minimax control problem. The optimal cost is $U_{0}\left(Q_{0}\right)$.

However, the formula (22) is not exactly dual to (17) in that it involves a sup $y$ instead of the expected $\mathbb{F}_{y}$. The problem stems from the fact that the cost to go $W_{t}$ is un-normalized. Introduce thus

$$
Q_{t}(x)=W_{t}(x)-\max _{\xi} W_{t}(\xi) .
$$

Now $Q_{t}$ is indeed a cost density. Let $\mathcal{Q}_{t}$ be the space of all possible such cost densities for every sequence $u_{[0, T-1]}$ and all $\omega$.

Introduce the set

$$
\mathrm{Y}_{t}(y)=\left\{(\xi, v) \in \mathbb{R}^{n} \times \mathbf{W} \mid h_{t}(\xi, v)=y\right\}=\bigcup_{x} \mathbf{Z}_{t}(x \mid u, y)
$$


Notice that although the rightmost set above seems to depend on $u$, it actually does not, since $u$ only enters the definition of $Z$ through the constraint $f_{t}(\xi, u, v)=x$. Let

$$
\widehat{Q}_{t+1}(x)=\max _{(\xi, v) \in \mathrm{Z}_{t}\left(x \mid u_{t}, y_{t}\right)}\left[Q_{t}(\xi)+\Gamma_{t}(v)\right]
$$

and

$$
\Lambda_{t}(y)=\max _{(\xi, w) \in \mathrm{Y}_{t}(y)}\left[Q_{t}(\xi)+\Gamma_{t}(w)\right] .
$$

Because of the last representation formula fo $Y_{t}(y)$ in (23), we have

$$
\max _{x} \widehat{Q}_{t+1}(x)=\Lambda_{t}\left(y_{t}\right)
$$

It follows that

$$
Q_{t+1}(x)=\widehat{Q}_{t+1}(x)-\Lambda_{t}\left(y_{t}\right)=: \mathcal{G}_{t}\left(Q_{t}, u_{t}, y_{t}\right)(x),
$$

where $\widehat{Q}_{t+1}$ and $\Lambda_{t}$ are defined by (24) and (25) respectively.

Moreover, according to the formula 1 for induced cost measures, $\Lambda_{t}$ is the cost measure induced on $y_{t}$ by $Q_{t}$ and $\Gamma_{t}$, i.e. the conditional cost measure given the past information $\left(u^{t-1}, y^{t-1}\right)$.

Then, we can now derive the analoguous formula to (14). For ease of notations, let in that derivation $Q_{t+1}[y]=\mathcal{G}\left(Q_{t}, u_{t}, y\right)$. For a given $u$, we need to compute for a test function $\psi(x)$

$$
\begin{aligned}
\mathbb{F}_{y} \mathbb{F}_{x}^{Q_{t+1}[y]} \psi(x)= & \\
\max _{y} & \left\{\max _{x}\left[\psi(x)+\max _{(\xi, w) \in \mathrm{Z}_{t}(x \mid u, y)}\left[Q_{t}(\xi)+\Gamma_{t}(w)\right]-\Lambda_{t}(y)\right]+\Lambda_{t}(y)\right\} .
\end{aligned}
$$

The $\Lambda_{t}$ 's cancel out, and the last two max operations collapse into

$$
\mathbb{F}_{y} \mathbb{F}_{x}^{Q_{t+1}[y]} \psi(x)=\max _{y} \max _{(\xi, w) \in Y_{t}(y)}\left[\psi\left(f_{t}(\xi, u, w)\right)+Q_{t}(\xi)+\Gamma_{t}(w)\right],
$$

or finally

$$
\mathbb{F}_{y} \mathbb{F}_{x}^{Q_{t+1}[y]} \psi(x)=\max _{\xi, w}\left[\psi\left(f_{t}(\xi, u, w)\right)+Q_{t}(\xi)+\Gamma_{t}(w)\right],
$$

which is the required formula, that we may write

$$
\mathbb{F}_{y} \mathbb{F}_{x}^{Q_{t+1}} \psi(x)=\mathbb{F}_{x}^{Q_{t}} \mathbb{F}_{w}^{\Gamma_{t}} \psi\left(f_{t}\left(x, u_{t}, w\right)\right) .
$$

(Compare with formula (14).)

We then easily derive the following theorem:

THEOREM 4.2. If there exists a sequence of functions $\left\{U_{t}\right\}$ from $\mathcal{Q}_{t}$ into $\mathbb{R}$ satisfying the following recursion

$$
\forall Q \in \mathcal{Q}_{T}, \quad U_{T}(Q)=\mathbb{F}^{Q} M
$$

(29) $\forall t \in[0, T-1], \forall Q \in \mathcal{Q}_{t}, \quad U_{t}(Q)=\min _{\mathbb{F}_{y}} U_{t+1}\left(\mathcal{G}_{t}(Q, u, y)\right)$

then the optimum value of the payoff $H(\mu)$ is $U_{0}\left(Q_{0}\right)$. Furthermore, if the minimum is always reached in (29) above, the argument of the minimum, $\hat{\mu}_{t}(Q)$ constitutes an optimal control strategy. 
Proof. The proof is identical to that of the stochastic case, only replacing $\mathbb{E}$ by $\mathbb{F}$, and invoking equation (27) instead of (14).

\section{Separation theorem}

\subsection{Stochastic Separation theorem}

We are here in the stochastic setup. The performance criterion is $G, V_{t}$ stands for the full information stochastic Bellman function as introduced in subsection 4.1.1, and $P_{t}$ stands for the conditional state probability density.

We can state the following result.

Theorem 5.1. Let

$$
S_{t}(x, u):=\mathbb{E}_{w_{t}} V_{t+1}\left(f_{t}\left(x, u, w_{t}\right)\right) P_{t}(x) .
$$

If there exists a (decreasing) sequence of (positive) numbers $R_{t}$ with $R_{T}=0$ such that,

$$
\begin{aligned}
& \forall t \in[0, T-1], \forall u_{[0, T-1]} \in \mathbf{U}, \forall \omega \in \Omega, \\
& \qquad \int \min _{u} S_{t}(x, u) d x+R_{t}=\min _{u} \int S_{t}(x, u) d x+R_{t+1},
\end{aligned}
$$

then the optimal control is obtained by minimizing the conditional expectation of the full information Bellman return function, i.e. choosing a minimizing $u$ in the right hand side above.

PROOF. The proof relies on the following fact :

LEMMA 5.2. Under the hypothesis of the proposition, the function

$$
U_{t}(P)=\mathbb{E}_{x}^{P} V_{t}(x)+R_{t}
$$

satisfies the dynamic programming equations (16)(17).

Let us check the lemma. Assume that $\forall P_{t+1} \in \mathcal{P}_{t+1}$,

$$
U_{t+1}\left(P_{t+1}\right)=\mathbb{E}_{x}^{P_{t+1}} V_{t+1}(x)+R_{t+1}=\int V_{t+1}(x) P_{t+1}(x) d x+R_{t+1}
$$

and apply (17), using (14), to get

$$
U_{t}\left(P_{t}\right)=\min _{u} \int \mathbb{E}_{w_{t}} V_{t+1}\left(f_{t}\left(x, u, w_{t}\right)\right) P_{t}(x) d x+R_{t+1} .
$$

Using the hypothesis of the theorem and Bellman's equation for $V_{t}$, it comes

$$
U_{t}\left(P_{t}\right)=\int V_{t}(x) P_{t}(x) d x+R_{t},
$$

and the recursion relation holds.

The hypothesis of the theorem sounds in a large extent like wishful thinking. It holds, as easily checked, in the linear quadratic case. (In that case, symmetry properties result in the certainty equivalence theorem.) There is little hope to find other instances. We state it here to stress the parallel with the minimax case. 


\subsection{Minimax separation theorem}

This section is based upon $[7,8]$. Essentially the same result appeared independantly in [18].

We are now in the minimax setup. The performance criterion is $H, V_{t}$ stands for the full information Isaacs Value function as introduced in subsection 4.2.1, and $Q_{t}$ stands for the conditional state cost density.

TheOREM 5.3. Let

$$
S_{t}(x, u)=\mathbb{F}_{w_{t}} V_{t+1}\left(f_{t}(x, u, w)\right)+Q_{t}(x) .
$$

If there exists a (decreasing) sequence of numbers $R_{t}$, such that,

$$
\begin{aligned}
& \forall t \in[0, T-1], \forall u_{[0, T-1]} \in \mathbf{U}, \forall \omega \in \Omega, \\
& \quad \max _{x} \min _{u} S_{t}(x, u)+R_{t}=\min _{u} \max _{x} S_{t}(x, u)+R_{t+1},
\end{aligned}
$$

then the optimal control is obtained by minimizing the conditional worst cost, future cost being measured according to the full information Isaacs Value function, i.e. taking a minimizing $u$ in the right hand side above.

Proof. The proof relies on the following fact:

LEMMA 5.4. Under the hypothesis of the proposition, the function

$$
U_{t}(Q)=\mathbb{F}_{x}^{Q} V_{t}(x)+R_{t}
$$

satisfies the dynamic programming equations (28)(29).

Let us check the lemma. Assume that, $\forall Q_{t+1} \in \mathcal{Q}_{t+1}$,

$$
U_{t+1}\left(Q_{t+1}\right)=\mathbb{F}_{x}^{Q_{t+1}} V_{t+1}(x)+R_{t+1}=\max _{x}\left[V_{t+1}(x)+Q_{t+1}(x)\right]+R_{t+1}
$$

and apply (29), using (27), to get

$$
\begin{aligned}
U_{t}\left(Q_{t}\right) & =\min _{u}\left(\max _{x, w}\left[V_{t+1}\left(f_{t}(x, u, w)\right)+\Gamma_{t}(w)+Q_{t}(x)\right]+R_{t+1}\right) \\
& =\min _{u} \max _{x} S_{t}(x, u)+R_{t+1} .
\end{aligned}
$$

Then, using the hypothesis of the proposition and Isaacs equation for $V$, it comes

$$
U_{t}\left(Q_{t}\right)=\max _{x}\left[V_{t}(x)+Q_{t}(x)\right]+R_{t},
$$

thus establishing the recursion relation.

The hypothesis of the proposition is not as unrealistic as in the stochastic case. It is satisfied in the linear quadratic case, but more generally, it can be satisfied if $S$ is convex-concave, for instance, with $R_{t}=0$. Moreover, in that case, the same $u$ provides the minimum in both sides, yielding a certainty equivalence theorem. 


\subsection{An ABSTRACT FORMULATION}

It is known that in the stochastic control problem, some results, including derivation of the separation theorem, are more easily obtained using a more abstract formulation of the observation process, in terms of a family of $\sigma$ fields $\mathcal{Y}_{t}$ generated in the disturbance space. The axioms are that

- the brownian motion $w_{t}$ is adapted to the family $\mathcal{Y}_{t}$,

- the family $\mathcal{Y}_{t}$ is increasing.

The same approach can be pursued in the minimax case. Instead of an explicit observation through an output (3), one may define the observation process in the following way. To each pair $\left(u_{[0, T-1]}, \omega\right)$, the observation process associates a sequence $\left\{\Omega_{t}\right\}_{t \in[0, T]}$ of subsets of $\Omega$. The axioms are that, for any $\left(u_{[0, T-1]}, \omega\right)$, the corresponding family $\Omega_{t}$ satisfies the following properties.

- The process is consistant, i.e. $\forall t, \omega \in \Omega_{t}$.

- The process is strictly non anticipative, i.e. $\omega \in \Omega_{t} \Leftrightarrow \omega^{t-1} \in \Omega_{t}^{t-1}$ where $\Omega_{t}^{t-1}$ stands for the set of restrictions to $[0, t-1]$ of the elements of $\Omega_{t}$.

- The process is with complete recall: $\forall\left(u_{[0, T-1]}, \omega\right), \quad t<t^{\prime} \Rightarrow \Omega_{t} \supset \Omega_{t^{\prime}}$.

In the case considered above, we have

$$
\Omega_{t}=\Omega\left(\mathbb{R}^{n} \mid u^{t}, y^{t}\right)
$$

but the abstract formulation suffices, and allows one, for instance, to extend the minimax certainty equivalence principle to a variable end time problem. See [8] for a detailed derivation, and [26] for an application of (a continuous version of) theorem 2 to a free end time problem.

One may think of the subsets $\Omega_{t}$ as playing the role of the measurable sets of the $\sigma$-field $\mathcal{Y}_{t}$.

\section{Conclusion}

The present setup shows a remarkable parallel between the stochastic and the minimax case, fully exploiting "Quadrat's morphism" between probabilities and maximisation. This goes all the way to the more powerfull abstract formulation for the observation process, and to the separation theorem (which we therefore write as a singular).

We may make a further remark. In the linear quadratic theory, it has been thought that the minimax certainty equivalence theorem of [4] was less a "separation" theorem than in the stochastic case because the "filter" to compute $\hat{x}$ depends on the weighting matrices in the criterion. We see here that another viewpoint is possible, in which these weighting matrices are only the counterpart to the gaussian law of the disturbances in the stochastic case, that also enter the Kalman filter. Then the exceptional fact with the LQG case is that it be possible to add a (quadratic) integral term to the (quadratic) terminal payoff. 


\section{REFERENCES}

[1] M. Akian: Density of idempotent measures and large deviations, Research Report 2534, INRIA, 1995.

[2] M. Akian, J-P. Quadrat and M. Viot: Bellman Processes, 11th International Conference on Systems Analysis and Optimization, Sophia Antipolis, France, 1994.

[3] F. Baccelli, G. Cohen, J-P. Quadrat, G-J. Olsder: Synchronization and Linearity, Wiley, Chichester, U.K., 1992.

[4] T. Başar and P. Bernhard: $H_{\infty}$ Optimal Control and Related Minimax Design Problems, a Game Theory Approach, second edition, Birkhauser, Boston, 1995.

[5] J.A. Ball and J.W. Helton: $\mathcal{H}_{\infty}$-Control for Nonlinear Plants: Connections with Differential Games, 28th CDC, Tampa, Fla, 1989.

[6] J.A. Ball, J.W. Helton and M.L. Walker: $H_{\infty}$-Control For Nonlinear Systems with Output Feedback, IEEE Trans. Automatic Control, AC-38, 1993, 546-559.

[7] P. Bernhard: Sketch of a Theory of Nonlinear Partial Information Min-Max Control, Research Report 2020, INRIA, 1993.

[8] P. Bernhard: A Discrete Time Min-max Certainty Equivalence Principle, Systems and Control Letters, 24, 1995, 229-234.

[9] P. Bernhard: Expected Values, Feared Values and Optimal Control, in G-J. Olsder ed. : New Trends in Dynamic Games and Applications, Birkhauser, 1995.

[10] P. Bernhard and G. Bellec: On the Evaluation of Worst Case Design, with an Application to the Quadratic Synthesis Technique, 3rd IFAC symposium on Sensitivity, Adaptivity and Optimality, Ischia, Italy, 1973.

[11] P. Bernhard and A. Rapaport: Min-max Certainty Equivalence Principle and Differential Games, revised version of a paper presented at the Workshop on Robust Controller Design and Differential Games, UCSB, Santa Barbara, 1993, submitted for publication.

[12] G. Choquet: Theory of capacities, Annales de l'Institut Fourier, Grenoble, 5, 1955, 131-295.

[13] G. Didinsky, T. Başar, and P. Bernhard: Structural Properties of Minimax Policies for a Class of Differential Games Arising in Nonlinear $H_{\infty}$-control, Systems and Control Letters, 21, 1993, 433-441.

[14] P. Del Moral: Résolution particulaire des problèmes d'estimation et d'optimisation non linéaires, $\mathrm{PhD}$ dissertation, Université Paul Sabatier, Toulouse, 1994.

[15] P. Del Moral: Maslov Optimization Theory, Russian Journal of Mathematical Physics, to appear.

[16] A. Isidori: Feedbak Control of Nonlinear Systems, 1st ECC, Grenoble, 1991.

[17] A. Isidori and A. Astolfi: Disturbance Attenuation and $\mathcal{H}_{\infty}$ Control via Measurement Feedback in Nonlinear Systems, IEEE Trans. on Automatic Control, AC 37, 1992, 1283-1293.

[18] M. James: On the Certainty Equivalence Principle and the Optimal Control of Partially Observed Dynamic Games, IEEE Trans. on Automatic Control, AC 39, 1994, 2321-2324.

[19] M.R. James and J.S. Baras: Partially Observed Differential Games, Infinite Dimensional HJI Equations, and Nonlinear $H_{\infty}$ Control, to appear.

[20] M.R. James, J.S. Barras and R.J. Elliott: Output Feedback Risk Sensitive Control and Differential Games for Continuous Time Nonlinear Systems, 32nd CDC, San Antonio, 1993.

[21] M.R. James, J.S. Barras and R.J. Elliott: Risk Sensitive Control and Dynamic Games for Partially Observed Discrete Time Nonlinear Systems, IEEE Trans. on Automatic Control, AC-39, 1994, 780-792. 
[22] V.N. Kolokolstov and V.P. Maslov: The general form of the endomorphisms in the space of continuous functions with values in a numerical commutative semiring (with the operation $\oplus=\max$ ), Soviet Math. Dokl., 36, 1988, 55-59.

[23] V. Maslov: Méthodes opératorielles, Éditions MIR, Moscow, 1987.

[24] V. Maslov and S.N. Samborskii: Idempotent analysis, Advances in Soviet Mathematics, 13, AMS, Providence, 1992.

[25] J-P. Quadrat: Théorèmes asymptotiques en programmation dynamique, Compte Rendus de l'Académie des Sciences, 311, 1990, 745-748.

[26] A. Rapaport and P. Bernhard: Un jeu de poursuite-evasion avec connaissance imparfaite des coordonnées, International Symposium on Differential Games and Applications, St Jovite, 1994.

[27] A.J. van der Schaft: Nonlinear State Space $\mathcal{H}_{\infty}$ Control Theory, ECC 1993, Groningen.

[28] M. Viot: Chaines de Markov dans $\mathbb{R} \min +$, META2 seminar, INRIA, 1992, unpublished.

[29] P. Whittle: Risk sensitive linear quadratic gaussian control, Advances in Applied Probability, 13, 1981, 764-777.

(C) Société de Mathématiques Appliquées et Industrielles 